\title{
Incidence and Prevalence of Deep Vein Thrombosis Among Neurocritical Intensive Care Unit Patients
}

\author{
Aman Dolla, MD1; Matthew Vibbert, MD ${ }^{1,2}$ \\ ${ }^{1}$ Department of Neurology, Thomas Jefferson University, Philadelphia, PA \\ 2 Department of Neurological Surgery, Thomas Jefferson University, Philadelphia, PA
}

Background: Deep venous thrombosis (DVT) of the lower extremities is a common cause of morbidity and mortality among neurologically injured patients. The data on incidence and prevalence rates of DVT among high risk neurologic populations is scarce. The available literature focuses largely on patients admitted to a medical or surgical intensive care unit with very limited information on patients in neuro-intensive care units (NICU). The aim of the present study is to assess the incidence and prevalence of deep vein thrombosis among patients admitted with acute neurologic injury.

Methods: We conducted a retrospective review of ultrasound records of 2,644 patients admitted to NICU, stroke or INICU at a university hospital over a 13-month period. We included all patients who underwent routine lower extremity ultrasound on admission and weekly. Data was abstracted and analyzed to assess the prevalence of DVT in this period. We excluded patients presenting with superficial vein thrombosis, hematoma and chronic venous scarring.

Results: Among the 2,644 patients studied, 161 were diagnosed with DVT. The overall prevalence of DVT was $6.1 \%$. Of the 161 cases of DVT; 87 (54\%) were diagnosed with DVT at the time of admission. In our sample, the rate of DVT present on admission was $3.3 \%$. There were 74 cases of hospital-acquired DVT, yielding an incidence of $2.8 \%$. Patients with DVT at the time of admission were largely Caucasian males with mean age 72 and mean SAPS II score of 34.2, ranging between 13 and 71.

Conclusions: Patients admitted to a neuro ICU are at high risk for having DVT present on admission and for acquiring DVT during their hospitalization. Further research is needed to understand the risk profile of patients with acute neurological injury. Asymptomatic screening of high risk patients on admission to a neurologic ICU, stroke unit, or intermediate care unit will identify a significant number of cases of DVT present of admission that might otherwise be misidentified as hospital acquired cases.

\section{INTRODUCTION}

Venous thromboembolism (VTE) including deep vein thrombosis (DVT) and pulmonary embolism (PE) is a significant health complication for critically ill hospitalized patients. DVT is a preventable health problem associated with several adverse outcomes including death. Among neurosurgical patients, the risk of DVT without prophylaxis is $22-35 \%$, while spinal cord injury patients who do not receive prophylaxis have rates ranging from $50-85 \% .{ }^{1}$ It is tempting to assume that previously healthy, acutely injured neuroscience patients who are diagnosed with DVT represent hospital-acquired disease; these patients generally do not have identifiable risk factors for DVT prior to the onset of acute illness. However, there is no data to support these assumptions.

Intensive care unit patients represent a heterogeneous population. The available literature describes the prevalence, incidence and risk factors associated with the development of DVT among patients admitted to medical and surgical intensive care units and following trauma, neurosurgery, or spinal cord injury. ${ }^{1-7}$ An estimated $10-30 \%$ of medical and surgical intensive care unit patients are known to develop DVT within the first week of admission. However, there is limited to no data about the DVT risk profile of patients admitted to a neuro-intensive care unit (NICU). The aim of the
Table 1. Prevalence and Incidence of DVT among acute neuroscience patients

\begin{tabular}{|l|l|}
\hline $\mathbf{N}$ & $\mathbf{2 6 4 4}$ \\
\hline Prevalence & $161(6.1 \%)$ \\
\hline Incidence & $74(2.9 \%)$ \\
\hline $\mathbf{N}$ & $\mathbf{1 6 1}$ \\
\hline DVT present on admission & $87(54.0 \%)$ \\
\hline DVT developed in hospital & $74(45.9 \%)$ \\
\hline
\end{tabular}

Table 2. Characteristics of patients with DVT at the time of admission

\begin{tabular}{|l|l|l|}
\hline & $\mathbf{N}$ & $\mathbf{8 7}$ \\
\hline \multirow{3}{*}{ Age (yrs) } & Mean \pm SD & $72 \pm 12.0$ \\
\cline { 2 - 3 } & Range & $29-94$ \\
\hline \multirow{3}{*}{ Sex } & Males & $52.7 \%$ \\
\cline { 2 - 3 } & Females & $47.3 \%$ \\
\hline \multirow{4}{*}{ Race } & Caucasian & $72.9 \%$ \\
\cline { 2 - 3 } & African-American & $12.2 \%$ \\
\cline { 2 - 3 } & Others & $1.5 \%$ \\
\hline \multirow{2}{*}{$\begin{array}{l}\text { SAPS II } \\
\text { score }\end{array}$} & Mean \pm SD & $34.2+14.8$ \\
\cline { 2 - 3 } & Range & $13-71$ \\
\hline
\end{tabular}

present study is to assess the incidence and prevalence of deep vein thrombosis among patients admitted with acute neurologic injury.

\section{METHODS}

In this retrospective study, data on all patients admitted to Neuro ICU, stroke or INICU of a tertiary care teaching hospital between December 2013 and January 2014 were included. Patients presenting with superficial vein thrombosis, hematoma and chronic venous scarring were excluded from the analysis. Our institution routinely conducts ultrasound screening within 24 hours of admission and weekly thereafter in high risk neurologically injured patients. 
A retrospective review of ultrasound records was conducted to identify patients with DVT.

To estimate the prevalence of DVT; number of patients presenting with DVT, identified through ultrasound screening within 24 hours of admission; patients with a history of chronic DVT and patients who developed DVT during their stay in the hospital identified through weekly ultrasound screening were computed. We estimated the overall prevalence of DVT by calculating the rate of DVT in our study population. We also describe the rate of DVT present on admission and the incidence of hospital-acquired DVT.

Various demographic characteristics, such as patient's age, sex, race were abstracted and health evaluation (SAPS II) score was calculated to describe the population presenting with DVT at the time of admission. Continuous data are expressed as mean with standard deviation and range. Categorical data are summarized to present counts and percentages.

\section{RESULTS}

A total of 2,644 ultrasound records were reviewed. Over a period of one year; the prevalence of DVT among neuro ICU patients was found to be $6.1 \%$, a total of 161 cases. Of the 161 patients diagnosed with DVT, 87 (54.0\%) presented with DVT at the time of admission. Patients developed DVT during their stay in the hospital in 74/161 (46\%) of all the cases diagnosed with DVT.

The study found that $3.3 \%$ of the 2,644 patients admitted to our acute neuroscience units between 2013-2014 presented with DVT at the time of admission. After excluding patients with a known case of DVT on admission, the population at risk of developing DVT in our sample comprised of 2,556 cases. The number of cases that developed DVT during their stay in the intensive care unit was 74 of 2,556 patients, resulting in an incidence rate of $2.9 \%$ among neuro ICU patients. (Table 1)

Analysis of various demographic characteristics revealed that majority of patients who presented with DVT on admission were Caucasian males with a mean age 72 . The mean SAPS II score for these cases was found to be 34.2. (Table 2)

\section{CONCLUSION}

There are several studies assessing the incidence and prevalence of DVT among critically ill surgical and medical ICU patients. Our study is among the few describing the prevalence of DVT among patients with acute neurologic injury. The prevalence of DVT among patients admitted to a neuro ICU is relatively high. Slightly over half of the cases diagnosed with DVT presented with this problem at the time of admission to the intensive care unit. Without timely admission screening, these cases will be incorrectly identified as hospitalacquired cases. Current best-practices such as early mobilization, mechanical compression devices, and chemoprophylaxis, remain important. These data suggest, however, that many patients acquire DVT within hours of their acute neurologic injury. Inpatient DVT risk prevention strategies are unlikely to impact the diagnosis of DVT for these patients. These patients may benefit from ultra-early, pre-hospital or emergency department-based interventions. In addition to the findings reported herein, further research is needed to understand the risk factors for ultra-early DVT and hospital acquired DVT within the high-risk population of patients with acute neurological injury.

\section{REFERENCES}

1. Attia J, Ray JG, Cook DJ, Douketis J, Ginsberg JS, Geerts WH. Deep vein thrombosis and its prevention in critically ill adults. Arch Intern Med. 2001; 161:1268-79.

2. Adriance SM, Murphy CV. Prophylaxis and treatment of venous thromboembolism in critically ill. International Journal of Critical Illness and Injury Science.2013; 3(2):143-151

3. Wilasrusmee $C$, Kiranantawat $K$, Horsirimanont S, Lertsithichai P, et.al. Deep vein thrombosis in surgical intensive care unit: Prevalence and Risk factors. Asian J Surg. 2009;32(2):85-8

4. Cook D, Crowther M, Meade M, Rabbat C, Griffith L, Schiff D, Geerts W, Guyatt G: Deep venous thrombosis in medical-surgical critically ill patients: prevalence, incidence, and risk factors. Crit Care Med. 2005, 33 (7):15651571.

5. Hong KC, Kim H, Kim JY, Kwak KS, Cho OM Cha HY, Lim SH, Song YJ: Risk factors and incidence of deep vein thrombosis in lower extremities among critically ill patients. J Clin Nurs. 2012, 21 (13-14): 1840-1846

6. Hirsch DR, Ingenito EP, Goldhaber SZ. Prevalence of deep venous thrombosis among patients in medical intensive care. JAMA 1995;274:336- 337.

7. Ibrahim EH, Iregui M, Prentice D, et al. Deep vein thrombosis during prolonged mechanical ventilation despite prophylaxis. Crit Care Med 2002;30:771-774. 